\title{
Continuous Erector Spinae Plane block with epidural catheter for post-operative analgesia following thoracic surgeries. A case series
}

\author{
L. Ali', A. Farooq ${ }^{1}$, A. Khan Raza², A. Taqi', I. Ali¹. \\ ${ }_{1}^{1}$ Doctors Hospital \& Medical Center Lahore, Department of Anaesthesia- intensive care and pain management, Lahore, Pakistan. \\ 2Doctors Hospital \& Medical Center, Department of Thoracic surgery, Lahore, Pakistan.
}

\section{Background \& Aims:}

Erector spinae plane block (ESPB) is a novel regional analgesia technique for thoracic and abdominal surgeries. It can be employed for analgesia as an alternative to epidural block in patients presenting for thoracic surgeries. ESPB provides adequate analgesia, is technically easier to perform, and safer due to lower risk of serious complications as compared to epidural block. We have illustrated this by presenting a case series of thoracic surgeries in which an ESPB catheter was used for postoperative analgesia. We reviewed our SOP regarding use of continuous ESPB use for thoracic surgeries.

\section{Methods:}

35 patients (ASA-III/IV) who underwent thoracic surgery (VATS, thoracotomy) under GA were included. Ultrasound-guided, 16-G epidural catheter was inserted at T5 level cephalo-caudally. 30 $\mathrm{mL}$ of $0.25 \%$ bupivacaine in ESPB administered prior to incision supplemented with Tramadol (1 $\mathrm{mg} / \mathrm{kg}$ IV). Postoperatively ESPB infusion was started at $10 \mathrm{~mL} / \mathrm{h}$ of $0.1 \%$ bupivacaine. The dose and catheter management in rooms/ward was done by anesthesia technicians. The outcomes assessed were number of hours in PACU stay, VAS 2 hourly in PACU and then 8 hourly in rooms/ward), safety profile and number of days of catheter in situ. Rescue analgesia was Tramadol $0.75 \mathrm{mg} / \mathrm{kg}$ IV SOS, Ketorolac $0.5 \mathrm{mg} / \mathrm{kg}$ IV BD (if not contraindicated) and Paracetamol $1 \mathrm{~g}$ IV TDS. The authors certify that appropriate consent were obtained from the patients and their identity is not been disclosed.

\section{No. of patients \& their VAS score}

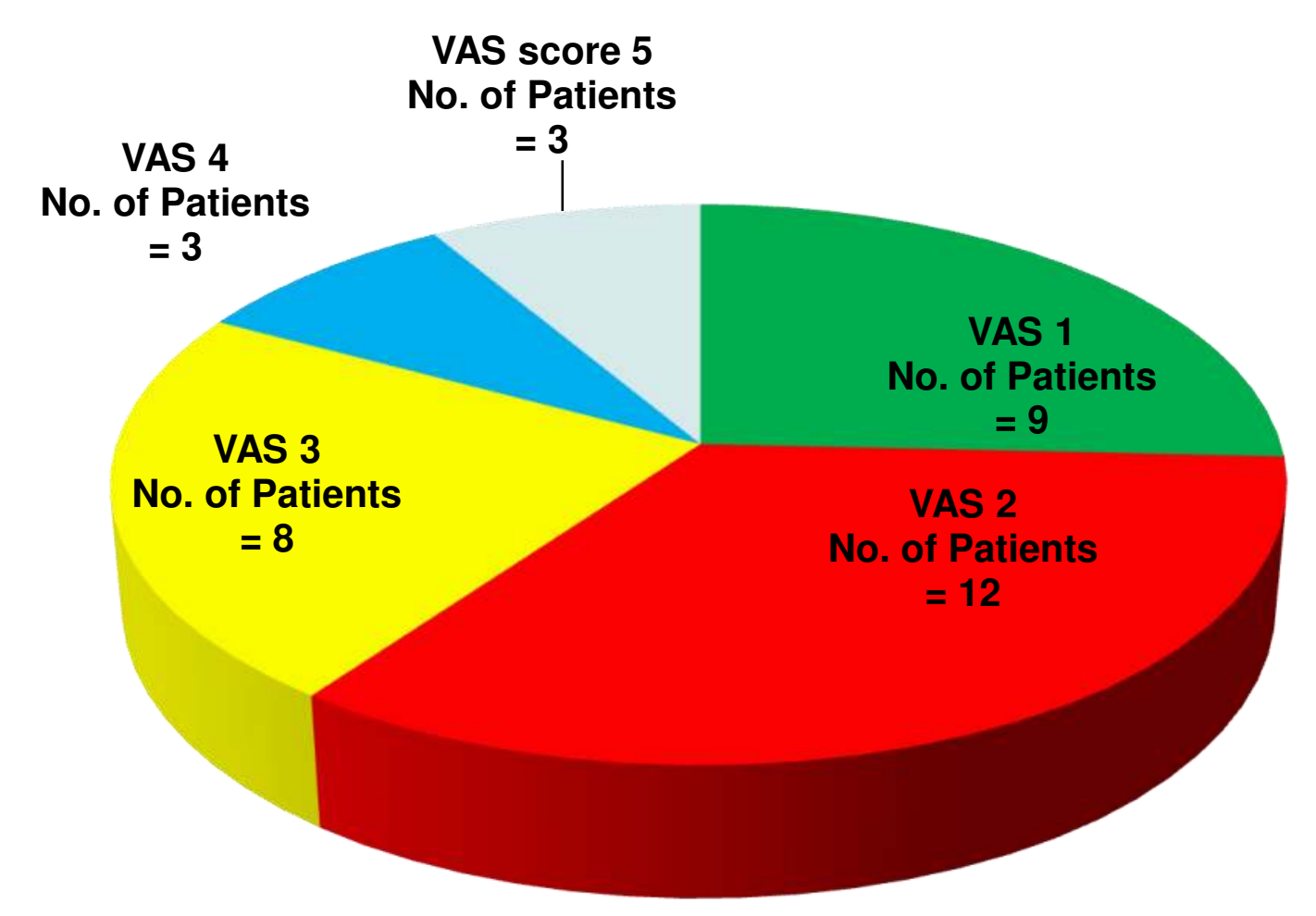

\section{Results:}

Patient stay in PACU was for $8 \pm 4$ hours. Mean VAS 2 hourly in PACU was $3 \pm 2$ while mean VAS 8 hourly in room/ward was $1 \pm 1$. None of the patients had hypotension necessitating vasopressor support, and the catheter remained in situ for $4 \pm 2$ days. $34.2 \%$ $(\mathrm{N}=12)$ patients who experienced VAS 5 , required supplemental analgesia.

\section{Discussion:}

TEA and PVB are mostly chosen as the first line regional analgesic techniques in thoracic surgeries for the pain management. ${ }^{[1,2]}$ When there is a contraindication or failure of these blocks, intercostal nerve block remained as an alternative but necessitating multiple injections. ESP block can serve as an alternative either as a single dose or as a continuous catheter based infusion for post-thoracotomy pain. Forero et al. [3] demonstrated ESPB as rescue analgesia in thoracotomy after a failed epidural. They revealed comparable pain score between TEA and ESP block until $12 \mathrm{~h}$ postextubation. The VAS scores remained to be persistently $\leq 4$ until $48 \mathrm{~h}$ in either of the group.

\section{Conclusions:}

Our case series reflects that ESPB provides adequate postoperative analgesia with no hemodynamic compromise in patients undergoing thoracic surgeries
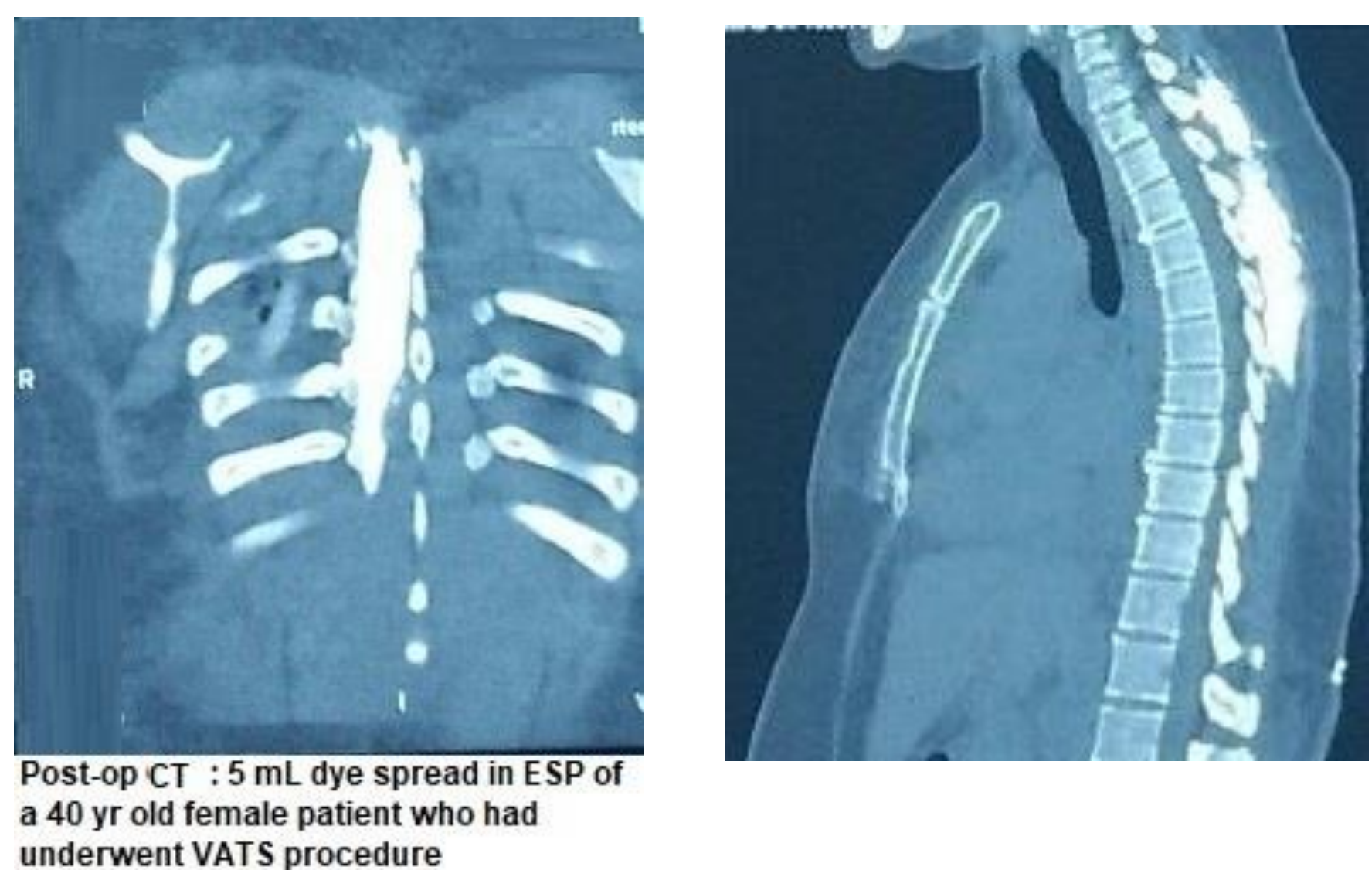

a 40 yr old female patient who ha underwent VATS procedure

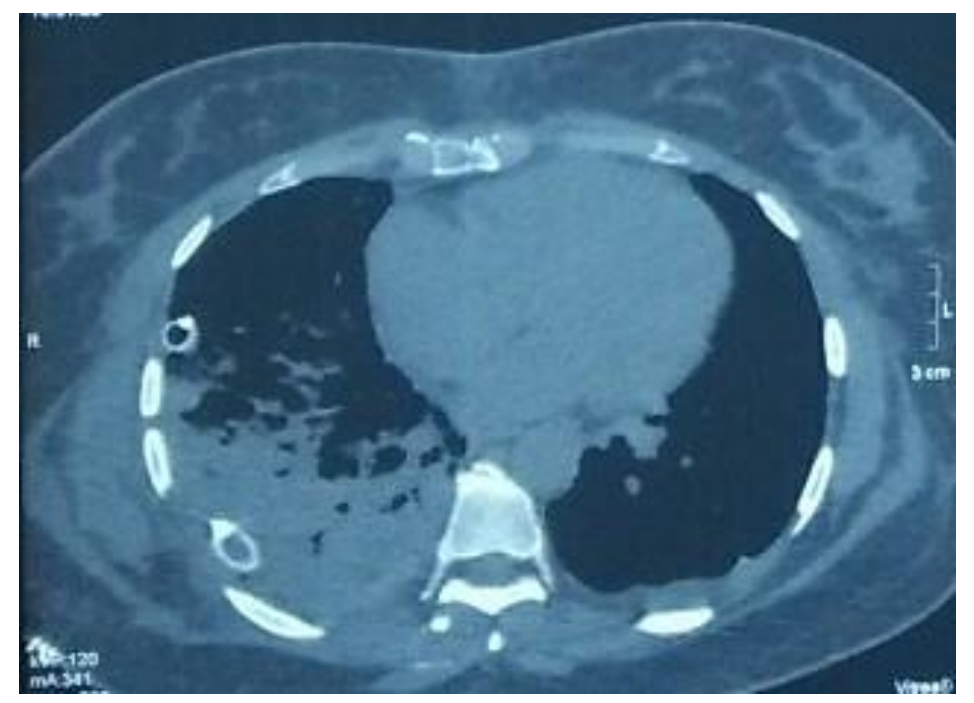

